\title{
DII-Based Linear Feedback Control Design for Practical Synchronization of Chaotic Systems with Uncertain Input Nonlinearity and Application to Secure Communication
}

\author{
Yeong-Jeu Sun \\ Department of Electrical Engineering, I-Shou University, Kaohsiung 840, Taiwan \\ Correspondence should be addressed to Yeong-Jeu Sun, yjsun@isu.edu.tw
}

Received 3 September 2012; Revised 31 October 2012; Accepted 5 November 2012

Academic Editor: Roberto Barrio

Copyright (C) 2012 Yeong-Jeu Sun. This is an open access article distributed under the Creative Commons Attribution License, which permits unrestricted use, distribution, and reproduction in any medium, provided the original work is properly cited.

\begin{abstract}
The concept of practical synchronization is introduced and the chaos synchronization of masterslave chaotic systems with uncertain input nonlinearities is investigated. Based on the differential and integral inequalities (DII) approach, a simple linear control is proposed to realize practical synchronization for master-slave chaotic systems with uncertain input nonlinearities. Besides, the guaranteed exponential convergence rate can be prespecified. Applications of proposed masterslave chaotic synchronization technique to secure communication as well as several numerical simulations are given to demonstrate the feasibility and effectiveness of the obtained result.
\end{abstract}

\section{Introduction}

Chaotic system is a kind of nonlinear dynamic system with unpredictable and irregular behavior. These characteristics may cause difficulties in controlling the system or may deteriorate the system performance. Besides, chaotic systems are extremely sensitive to their initial conditions, so that they are not readily synchronized. However, if these characteristics can be applied skillfully, there are some merits that may be utilized, for instance, applying the chaotic synchronization scheme to chaotic secure communications. On the other hand, linear feedback controllers have the following advantages over nonlinear controllers: (i) low implementation complexity; (ii) easily realized in hardware; (iii) reduced sensitivity to parameter variations; (iv) improved reference tracking performance [1-4]. Consequently, how to design a linear feedback controller instead of a complicated nonlinear controller is a main issue in the field of chaos synchronization. 
In recent years, a wide variety of methodologies in the synchronization of chaotic systems have been proposed, such as Lyapunov's stability theory, adaptive control approach, variable structure control (VSC) approach, $H_{\infty}$ control approach, adaptive sliding mode control approach, backstepping control approach, projective synchronization approach, timedomain approach, and others. For more detailed knowledge, one can refer to [5-12].

Over the past decades, generalized Lorenz systems, which are much more useful than traditional Lorenz system in practical applications, have been received a great deal of interest due to theoretical interests and successful applications in numerous areas; see, for example, [6, 8, 10, 12-15]. In [8], by means of linearization and Lyapunov's stability theory, a linear state error feedback control has been presented to guarantee the uniform chaos synchronization of master-slave identical generalized Lorenz systems without any uncertainties. In [14, 15], two kinds of state observers for the generalized Lorenz chaotic system have been developed to guarantee the global exponential stability of the resulting error system. Besides, based on the adaptive sliding mode control approach, a single nonlinear control has been proposed in [6] to ensure the synchronization of master-slave identical generalized Lorenz systems without any uncertainties. In [10], using Lyapunov's stability theory, a linear feedback controller has been developed to realize the exponential synchronization of master-slave identical generalized Lorenz systems without any uncertain input nonlinearity. Meanwhile, some control strategies have been established in [12] to guarantee the coexistence of antiphase and complete synchronization in master-slave identical generalized Lorenz systems without any uncertainties. Besides, based on the time domain approach, the upper solution bound and lower solution bound of the generalized Lorenz chaotic system have been offered in [13].

Owing to unavoidable tolerances and uncontrollable and unpredictable environmental conditions, it seems to be difficult and impossible to maintain the parameter values (e.g., resistors, inductors, and capacitors) of the controllers as fixed values. Therefore, uncertain input nonlinearity always exists in dynamic control systems. Over the past decades, researchers have been concerned with various uncertain input nonlinearities common in nonlinear systems, such as deadzones, saturation, hysteresis, relays, and others; see, for instance, $[5,7,9,16-20]$ and the references therein.

In this paper, motivated by the discussion mentioned above, the chaos synchronization of master-slave identical generalized Lorenz systems with uncertain input nonlinearities will be investigated. Using the DII methodology, a linear feedback control is proposed to realize practical synchronization for such master-slave systems with any prespecified exponential convergence rates. Applications of proposed master-slave chaotic synchronization technique to secure communication as well as several numerical simulations are given to demonstrate the feasibility and effectiveness of the obtained result.

This paper is organized as follows. The problem formulation and main result are presented in Section 2. Several numerical simulations are given in Section 3 to illustrate the main result. Finally, conclusion is made in Section 4 . Note that throughout the remainder of this paper, the notation $A^{T}$ is used to denote the transpose for a matrix $A$, and $\|x\|:=\sqrt{x^{T} \cdot x}$ denotes the Euclidean norm of the column vector $x$.

\section{Problem Formulation and Main Result}

In this paper, we consider the following master-slave chaotic systems with uncertain input nonlinearities. 
Master system is as follows:

$$
\begin{gathered}
\dot{x}_{1}(t)=\left(10+\frac{25}{29} a\right) \cdot\left[x_{2}(t)-x_{1}(t)\right], \\
\dot{x}_{2}(t)=\left(28-\frac{35}{29} a\right) x_{1}(t)+(a-1) x_{2}(t)-x_{1}(t) x_{3}(t), \\
\dot{x}_{3}(t)=\left(-\frac{8}{3}-\frac{1}{87} a\right) x_{3}(t)+x_{1}(t) x_{2}(t), \\
{\left[\begin{array}{lll}
x_{1}(0) & x_{2}(0) & x_{3}(0)
\end{array}\right]^{T}=\left[\begin{array}{lll}
\Delta x_{10} & \Delta x_{20} & \Delta x_{30}
\end{array}\right]^{T},}
\end{gathered}
$$

slave system is as follows:

$$
\begin{gathered}
\dot{z}_{1}(t)=\left(10+\frac{25}{29} a\right) \cdot\left[z_{2}(t)-z_{1}(t)\right]+\Delta \phi_{1}\left(u_{1}\right) \\
\dot{z}_{2}(t)=\left(28-\frac{35}{29} a\right) z_{1}(t)+(k-1) z_{2}(t)-z_{1}(t) z_{3}(t)+\Delta \phi_{2}\left(u_{2}\right) \\
\dot{z}_{3}(t)=\left(-\frac{8}{3}-\frac{1}{87} a\right) z_{3}(t)+z_{1}(t) z_{2}(t)+\Delta \phi_{3}\left(u_{3}\right) \\
{\left[\begin{array}{lll}
z_{1}(0) & z_{2}(0) & z_{3}(0)
\end{array}\right]^{T}=\left[\begin{array}{lll}
\Delta z_{10} & \Delta z_{20} & \Delta z_{30}
\end{array}\right]^{T}}
\end{gathered}
$$

where $x(t):=\left[\begin{array}{lll}x_{1}(t) & x_{2}(t) & x_{3}(t)\end{array}\right]^{T} \in \mathfrak{R}^{3 \times 1}$ and $z(t):=\left[\begin{array}{lll}z_{1}(t) & z_{2}(t) & z_{3}(t)\end{array}\right]^{T} \in \mathfrak{R}^{3 \times 1}$ are state vectors, $a$ is the system parameter with $0 \leq a<1,\left[\begin{array}{lll}\Delta x_{10} & \Delta x_{20} & \Delta x_{30}\end{array}\right]^{T}$ is the unknown initial value satisfying $\|x(0)\| \leq R$, where $R \geq 0$ is given, $u=\left[\begin{array}{lll}u_{1} & u_{2} & u_{3}\end{array}\right]^{T} \in \mathfrak{R}^{3}$ is the control input, and $\Delta \phi_{i}(\cdot): \mathfrak{R} \rightarrow \mathfrak{R}$ is the uncertain input nonlinearity for every $i \in\{1,2,3\}$. It is noted that the system (2.1a)-(2.1d), displays chaotic behavior for each $0 \leq a<1$ [21]. The original Lorenz system is a special case of system (2.1a)-(2.1d), with $a=0$. The objective of this paper is to search a tracking control law $u=\left[\begin{array}{lll}u_{1} & u_{2} & u_{3}\end{array}\right]^{T} \in \mathfrak{R}^{3}$ such that the states $z_{1}$, $z_{2}$, and $z_{3}$ of the slave system (2.2a)-(2.2d) track, respectively, the states $x_{1}, x_{2}$, and $x_{3}$ of the master system (2.1a)-(2.1d), with any desired exponential convergence rate.

Throughout this paper, the following assumption is made:

(A1) There exist positive numbers $\beta_{1}, \beta_{2}$, and $\beta_{3}$ such that

$$
\beta_{i} \cdot u_{i}^{2} \leq u_{i} \Delta \phi\left(u_{i}\right), \quad \forall i \in\{1,2,3\}
$$

Remark 2.1. Generally speaking, if the uncertain input nonlinearity satisfies

$$
r_{1} \cdot u^{2} \leq u \Delta \phi(u) \leq r_{2} \cdot u^{2}, \quad \forall u \in \Re,
$$

we often refer $r_{2}$ as the gain margin and $r_{1}$ as the gain reduction tolerance. 
For brevity, let us define the synchronous error vector as

$$
e(t):=\left[\begin{array}{lll}
e_{1}(t) & e_{2}(t) & e_{3}(t)
\end{array}\right]^{T}:=z(t)-x(t) .
$$

The precise definition of practical synchronization is given as follows.

Definition 2.2. Given any $\alpha>0$ and $R \geq 0$, the slave system (2.2a)-(2.2d) practically synchronizes the master system $(2.1 \mathrm{a})-(2.1 \mathrm{~d})$ provided that there exist a suitable control $u(e, \alpha, R)$ and a positive number $k_{1}$ such that the following conditions are satisfied:

(i) the synchronous error satisfies $\|e(t)\| \leq k_{1} \cdot e^{-\alpha t}, \forall t \geq 0$;

(ii) the control law of $u(e, \alpha, R)$ is linear in the synchronous error $e$.

In this case, the positive number $\alpha$ is called the exponential convergence rate. In other words, the practical synchronization means that there exists a linear control law such that the state of the slave system can track the state of the master system with any desired exponential convergence rate.

Now we present the main result for the practical synchronization between system (2.1a) $-(2.1 \mathrm{~d})$ and system $(2.2 \mathrm{a})-(2.2 \mathrm{~d})$.

Theorem 2.3. The uncertain slave system (2.2a)-(2.2d) practically synchronizes the master system (2.1a)-(2.1d) with the exponential convergence rate $\alpha$, under the linear feedback control

$$
u(e, \alpha, R)=-k e(t),
$$

where

$$
\begin{aligned}
& k=\frac{(\alpha+(\sqrt{2} / 2) p)}{\beta}, \\
& p \geq \max \left\{r_{1}, r_{2}\right\}, \\
& \beta=\min \left\{\beta_{1}, \beta_{2}, \beta_{3}\right\}, \\
& r_{1}:=\frac{((8 / 3)+(a / 87)) \cdot(19-(5 a / 29))}{\sqrt{(1-a) \cdot(5 / 3+88 a / 87)}}, \\
& r_{2}:=R+38-\frac{10 a}{29} .
\end{aligned}
$$

Proof. From Theorem 1 of [13], one has

$$
\begin{aligned}
& \min \left\{-r_{1},-\sqrt{p_{2}}\right\} \leq x_{2}(t) \leq \max \left\{r_{1}, \sqrt{p_{2}}\right\}, \quad \forall t \geq 0, \\
& \min \left\{-r_{1}+38-\frac{10 a}{29},-\sqrt{p_{2}}+38-\frac{10 a}{29}\right\} \\
& \quad \leq x_{3}(t) \leq \max \left\{r_{1}+38-\frac{10 a}{29}, \sqrt{p_{2}}+38-\frac{10 a}{29}\right\}, \quad \forall t \geq 0,
\end{aligned}
$$


with

$$
p_{2}=\Delta x_{10}^{2}+\Delta x_{20}^{2}+\left(\Delta x_{30}-38+\frac{10 a}{29}\right)^{2}
$$

This implies that

$$
\begin{gathered}
\left|x_{2}(t)\right| \leq \max \left\{r_{1}, r_{2}\right\} \leq p, \quad \forall t \geq 0, \\
\left|38-\frac{10 k}{29}-x_{3}(t)\right| \leq \max \left\{r_{1}, r_{2}\right\} \leq p, \quad \forall t \geq 0,
\end{gathered}
$$

in view of $\|x(0)\| \leq R$ and $\sqrt{p_{2}} \leq p$. From (2.1a)-(2.1d)-(2.5), we deduce that, for every $t \geq 0$,

$$
\begin{aligned}
\dot{e}_{1} & =\left(10+\frac{25}{29} a\right) \cdot\left(e_{2}-e_{1}\right)+\Delta \phi_{1}\left(u_{1}\right) \\
\dot{e}_{2}(t) & =\left(28-\frac{35}{29} a\right) e_{1}+(a-1) e_{2}-e_{1} e_{3}-x_{1} e_{3}-x_{3} e_{1}+\Delta \phi_{2}\left(u_{2}\right), \\
\dot{e}_{3} & =\left(-\frac{8}{3}-\frac{1}{87} a\right) e_{3}+e_{1} e_{2}+x_{2} e_{1}+x_{1} e_{2}+\Delta \phi_{3}\left(u_{3}\right) .
\end{aligned}
$$

Let

$$
W(t):=e_{1}^{2}(t)+e_{2}^{2}(t)+e_{3}^{2}(t)
$$

The time derivative of $W(t)$ along the trajectories of the closed-loop system (2.15a)-(2.15c) with (2.6)-(2.10) is given by

$$
\begin{aligned}
\frac{d W(t)}{d t}= & 2 e_{1}\left(10+\frac{25 a}{29}\right)\left(e_{2}-e_{1}\right)+2 e_{1} \Delta \phi_{2}\left(u_{1}\right) \\
& +2 e_{2}\left[\left(28-\frac{35 a}{29}\right) e_{1}+(a-1) e_{2}-e_{1} e_{3}\right] \\
& +2 e_{2}\left(-x_{1} e_{3}-x_{3} e_{1}+\Delta \phi_{1}\left(u_{2}\right)\right)+2 e_{3}\left[\left(\frac{-8}{3}-\frac{a}{87}\right) e_{3}+e_{1} e_{2}\right] \\
& +2 e_{3}\left(x_{2} e_{1}+x_{1} e_{2}+\Delta \phi_{3}\left(u_{3}\right)\right)
\end{aligned}
$$




$$
\begin{aligned}
= & -2\left(10+\frac{25 a}{29}\right) e_{1}^{2}-2(1-a) e_{2}^{2}-2\left(\frac{8}{3}+\frac{a}{87}\right) e_{3}^{2} \\
& +2\left(38-\frac{10 a}{29}-x_{3}\right) e_{1} e_{2}+2 x_{2} e_{1} e_{3}-\sum_{i=1}^{3} \frac{2 \beta}{(\alpha+(\sqrt{2} / 2) p)} u_{i} \Delta \phi_{i}\left(u_{i}\right) \\
\leq & 2\left(10+\frac{25 k}{29}\right) e_{1}^{2}-2(1-k) e_{2}^{2}-2\left(\frac{8}{3}+\frac{k}{87}\right) e_{3}^{2} \\
& +2\left(38-\frac{10 k}{29}-x_{3}\right) e_{1} e_{2}+2 x_{2} e_{1} e_{3}-\sum_{i=1}^{3} \frac{2 \beta}{(\alpha+(\sqrt{2} / 2) p)} \beta_{i} u_{i}^{2} \\
= & -2\left(10+\frac{25 a}{29}\right) e_{1}^{2}-2(1-a) e_{2}^{2}-2\left(\frac{8}{3}+\frac{a}{87}\right) e_{3}^{2} \\
& +2\left(38-\frac{10 a}{29}-x_{3}\right) e_{1} e_{2}+2 x_{2} e_{1} e_{3}-\sum_{i=1}^{3} \frac{2 \beta_{i}}{\beta}\left(\alpha+\frac{\sqrt{2}}{2} p\right) e_{i}^{2} \\
\leq & -2\left(10+\frac{25 a}{29}\right) e_{1}^{2}-2(1-a) e_{2}^{2}-2\left(\frac{8}{3}+\frac{a}{87}\right) e_{3}^{2} \\
& +2\left(38-\frac{10 a}{29}-x_{3}\right) e_{1} e_{2}+2 x_{2} e_{1} e_{3}-\sum_{i=1}^{3}(2 \alpha+\sqrt{2} p) e_{i}^{2} \\
\leq & 2\left|38-\frac{10 a}{29}-x_{3}\right| \cdot\left|e_{1}\right| \cdot\left|e_{2}\right|+2\left|x_{2}\right|\left|e_{1}\right|\left|e_{3}\right|-(2 \alpha+\sqrt{2} p)\left(e_{1}^{2}+e_{2}^{2}+e_{3}^{2}\right) \\
\leq & 2 p \cdot\left|e_{1}\right| \cdot\left|e_{2}\right|+2 p\left|e_{1}\right|\left|e_{3}\right|-(2 \alpha+\sqrt{2} p)\left(e_{1}^{2}+e_{2}^{2}+e_{3}^{2}\right) \\
\leq & \frac{\sqrt{2}}{2} p \cdot e_{1}^{2}+\sqrt{2} p \cdot e_{2}^{2}+\frac{\sqrt{2}}{2} p \cdot e_{1}^{2}+\sqrt{2} p \cdot e_{3}^{2}-(2 \alpha+\sqrt{2} p)\left(e_{1}^{2}+e_{2}^{2}+e_{3}^{2}\right) \\
= & -2 \alpha W(t), \forall t \geq 0,
\end{aligned}
$$

in view of (2.14). It is easy to deduce that

$$
e^{2 \alpha t} \cdot \dot{W}(t)+e^{2 \alpha t} \cdot 2 \alpha W(t)=\frac{d}{d t}\left[e^{2 \alpha t} \cdot W(t)\right] \leq 0, \quad \forall t \geq 0
$$

It follows that

$$
\int_{0}^{t} \frac{d}{d t}\left[e^{2 \alpha t} \cdot W(t)\right] d t=e^{2 \alpha t} \cdot W(t)-W(0) \leq \int_{0}^{t} 0 d t=0, \quad \forall t \geq 0 .
$$

Thus, from (2.16) and (2.19), it can be readily obtained that

$$
\|e(t)\|^{2}=W(t) \leq e^{-2 \alpha t} W(0), \quad \forall t \geq 0 .
$$


Consequently, we conclude that

$$
\|e(t)\| \leq e^{-\alpha t} \sqrt{W(0)}, \quad \forall t \geq 0
$$

This completes the proof.

Remark 2.4. Based on the adaptive sliding mode control approach, a single nonlinear control has been proposed in [6] to realize the synchronization of master-slave identical generalized Lorenz systems without any uncertainties. It is seen that our designed control (2.6) is a simple linear form which is much simpler than the nonlinear form proposed in [6]. Obviously, the proposed linear feedback control form is much more simply implemented.

Remark 2.5. In this paper, the merits of DII approach can be stated as follows.

(i) Based on the DII approach, the proposed control law has certain intrinsic robustness properties, in particular, infinite gain margin.

(ii) Based on the DII approach, the proposed feedback control can be easily implemented owing to the linearity of (2.6).

(iii) Based on the DII approach, not only the exponential synchronization can be realized but also the guaranteed exponential convergence rate can be prespecified.

Remark 2.6. In what follows, we present an algorithm to find the linear control law of (2.6) stated in Theorem 2.3.

\section{INPUT}

The master-slave chaotic systems with uncertain input nonlinearities (2.1a)-(2.1d)-(2.2a)(2.2d) the parameters $a, \alpha$, and $R$.

\section{OUPUT}

linear control of (2.6).

Step 1. Choose $\beta_{1}, \beta_{2}$, and $\beta_{3}$ such that (A1) is satisfied.

Step 2. Determine $\beta$ from (2.9).

Step 3. Determine $r_{1}$ and $r_{2}$ from (2.10) and (2.11).

Step 4. Determine $p$ from (2.8).

Step 5. Determine $k$ from (2.7).

Step 6. OUPUT $u(e, \alpha, R)=-k e(t)$. 


\section{Numerical Examples and Simulations}

In what follows, we provide two examples to illustrate the main results.

Example 3.1. Consider the uncertain master-slave systems (2.1a)-(2.1d), and (2.2a)-(2.2d) with $a=0.4, R=8$, and uncertain input nonlinearities:

$$
\Delta \phi_{1}\left(u_{1}\right)=\Delta a_{1} \cdot u_{1}+\Delta b_{1} u_{1}^{3}, \quad \Delta \phi_{2}\left(u_{2}\right)=\left(\Delta a_{2}+\Delta b_{2} \sin t\right) \cdot u_{2}, \quad \Delta \phi_{3}\left(u_{3}\right)=\Delta a_{3} \cdot u_{3} .
$$

In addition, the uncertain parameters are bounded by

$$
\Delta b_{1} \geq 0, \quad\left|\Delta b_{2}\right| \leq 1, \quad 5 \leq \Delta a_{i}, \quad \forall i \in\{1,2,3\} .
$$

Comparison of (3.1a) and (3.1b) with (A1) and (2.9) yields

$$
\beta_{1}=\beta_{3}=5, \quad \beta_{2}=\beta=4 \text {. }
$$

From (2.8), (2.10), and (2.11), one has

$$
r_{1}=45.35, \quad r_{2}=45.86, \quad p=45.9 \geq 45.86=\max \left\{r_{1}, r_{2}\right\}
$$

Furthermore, from (2.7), it is easy to deduce that

$$
k=0.25 \alpha+8.11
$$

Thus, we obtain the design controller

$$
u(e, \alpha, R)=-(0.25 \alpha+8.11) \cdot e(t)
$$

in view of (2.6). Consequently, by Theorem 2.3, we conclude that the system (2.2a)-(2.2d) with the linear control (3.5) practically synchronizes the generalized Lorenz chaotic system (2.1a)-(2.1d), with the guaranteed exponential convergence rate $\alpha$.

The typical state trajectories of the sytem (2.1a)-(2.1d) with $a=0.4$, are depicted in Figure 1. Furthermore, the synchronization errors of systems (2.1a)-(2.1d), and (2.2a)(2.2d) with the linear control (3.5) are depicted in Figure 2. From the foregoing simulation 


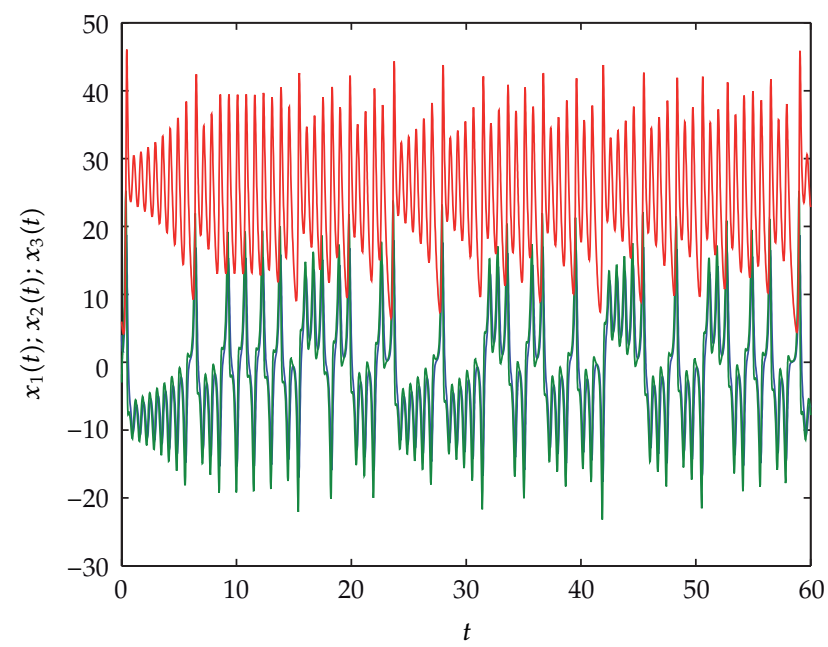

Figure 1: Typical state trajectories of the system (2.1a)-(2.1d) with $a=0.4$.

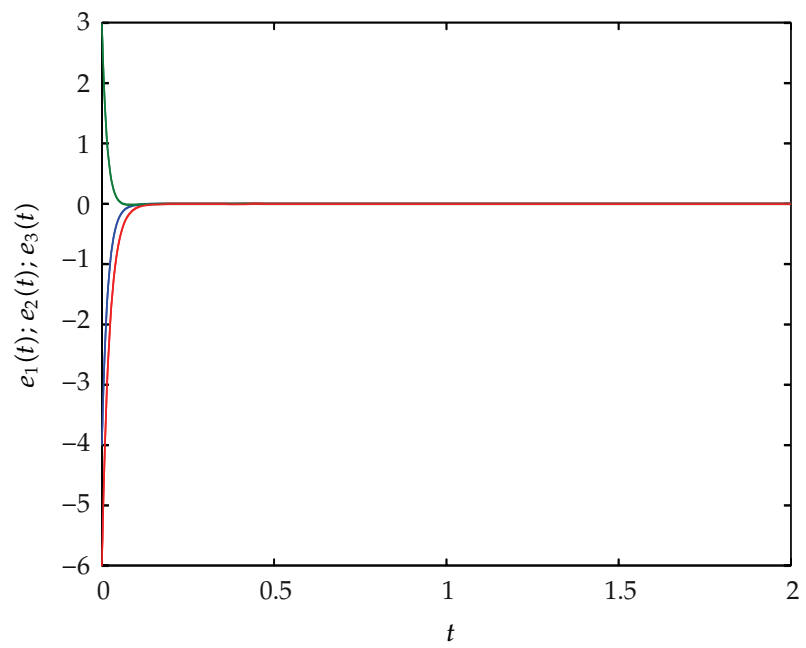

Figure 2: Synchronization errors of systems (2.1a)-(2.1d) and (2.2a)-(2.2d) with $a=0.4$ and $\alpha=1$.

results, it is seen that the controlled uncertain master-slave systems (2.1a)-(2.1d) and (2.2a)(2.2d) realize the practical synchronization under the linear control (3.5). It is noted that [8] has proposed a linear control to achieve the synchronization of the systems (2.1a)-(2.1d) and $(2.2 \mathrm{a})-(2.2 \mathrm{~d})$ without any uncertain input nonlinearity, but the design control only guarantees that the synchronization error system is asymptotically stable. The comparisons of the error systems' trajectories are shown in Figures 3 and 4.

Example 3.2. Consider the following secure communication system and the proposed scheme is illustrated in Figure 5. 


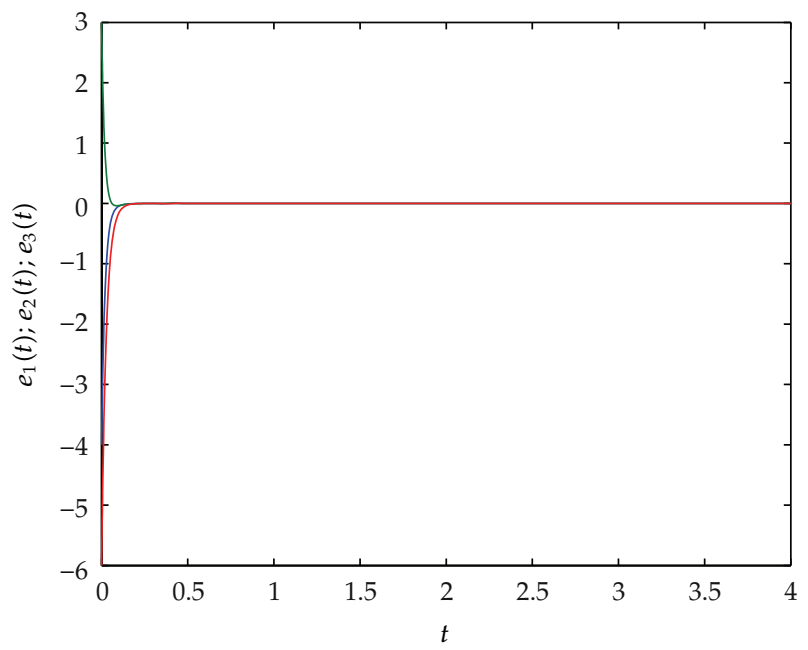

Figure 3: Synchronization errors of systems (2.1a)-(2.1d) and (2.2a)-(2.2d) with $a=0.4$ and $\Delta \phi_{i}\left(u_{i}\right)=$ $u_{i}, \forall i \in\{1,2,3\}$, under the control of (2.6).

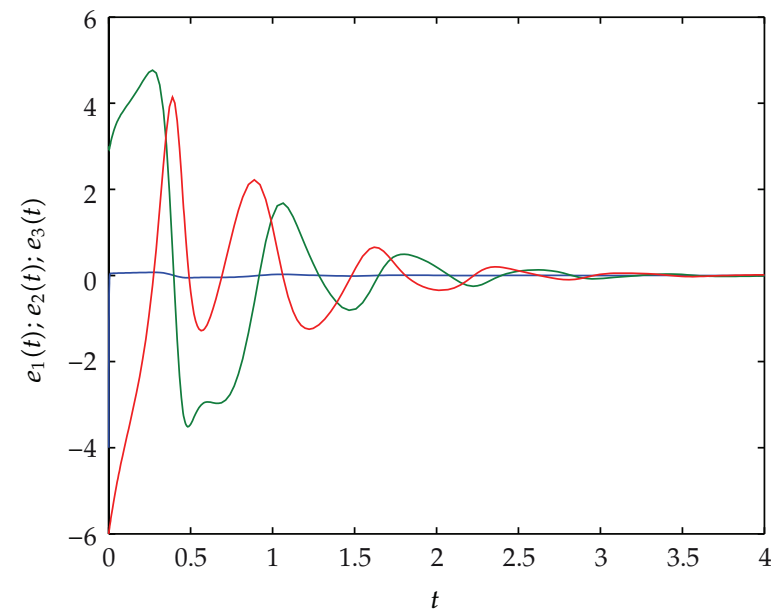

Figure 4: Synchronization errors of systems (2.1a)-(2.1d) and (2.2a)-(2.2d), with $a=0.4$ and $\Delta \phi_{i}\left(u_{i}\right)=$ $u_{i}, \forall i \in\{1,2,3\}$, under the control proposed in [8].

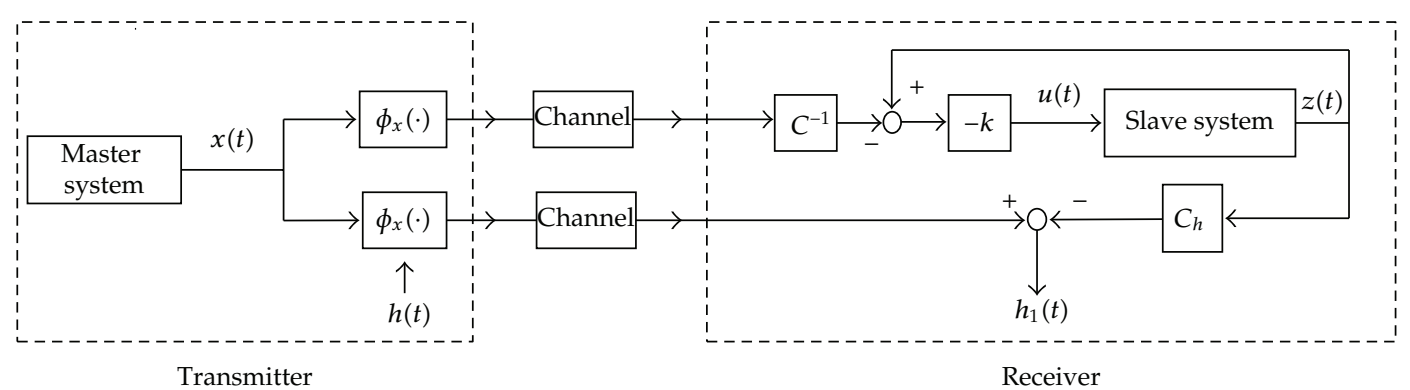

Figure 5: Secure communication system. 
Transmitter is as follows:

$$
\begin{gathered}
\dot{x}_{1}(t)=\left(10+\frac{25}{29} a\right) \cdot\left[x_{2}(t)-x_{1}(t)\right], \\
\dot{x}_{2}(t)=\left(28-\frac{35}{29} a\right) x_{1}(t)+(a-1) x_{2}(t)-x_{1}(t) x_{3}(t), \\
\dot{x}_{3}(t)=\left(-\frac{8}{3}-\frac{1}{87} a\right) x_{3}(t)+x_{1}(t) x_{2}(t), \\
\phi_{x}(t)=C x(t), \\
\phi_{h}(t)=C_{h} x(t)+h(t), \\
{\left[\begin{array}{lll}
x_{1}(0) & x_{2}(0) & x_{3}(0)
\end{array}\right]^{T}=\left[\begin{array}{lll}
\Delta x_{10} & \Delta x_{20} & \Delta x_{30}
\end{array}\right]^{T},}
\end{gathered}
$$

Receiver is as follows:

$$
\begin{gathered}
\dot{z}_{1}(t)=\left(10+\frac{25}{29} a\right) \cdot\left[z_{2}(t)-z_{1}(t)\right]+\Delta \phi_{1}\left(u_{1}\right), \\
\dot{z}_{2}(t)=\left(28-\frac{35}{29} a\right) z_{1}(t)+(k-1) z_{2}(t)-z_{1}(t) z_{3}(t)+\Delta \phi_{2}\left(u_{2}\right), \\
\dot{z}_{3}(t)=\left(-\frac{8}{3}-\frac{1}{87} a\right) z_{3}(t)+z_{1}(t) z_{2}(t)+\Delta \phi_{3}\left(u_{3}\right), \\
h_{1}(t)=\phi_{h}(t)-C_{h} z(t), \\
{\left[\begin{array}{lll}
z_{1}(0) & z_{2}(0) & z_{3}(0)
\end{array}\right]^{T}=\left[\begin{array}{lll}
\Delta z_{10} & \Delta z_{20} & \Delta z_{30}
\end{array}\right]^{T,}}
\end{gathered}
$$

where $u$ is designed as (2.6)-(2.11), $x(t) \quad:=\left[\begin{array}{lll}x_{1}(t) & x_{2}(t) & x_{3}(t)\end{array}\right]^{T} \in \mathfrak{R}^{3 \times 1}$, $z(t):=\left[\begin{array}{lll}z_{1}(t) & z_{2}(t) & z_{3}(t)\end{array}\right]^{T} \in \mathfrak{R}^{3 \times 1},\left[\begin{array}{lll}\Delta x_{10} & \Delta x_{20} & \Delta x_{30}\end{array}\right]^{T}$ is the unknown initial value satisfying $\|x(0)\| \leq 8, C \in \mathfrak{R}^{3 \times 3}$ is a nonsingular matrix, $h(t) \in \mathfrak{R}^{q \times 1}$ is the information vector, $C_{h} \in \mathfrak{R}^{q \times 3}, h_{1}(t) \in \mathfrak{R}^{q \times 1}$ is the signal recovered from $h(t)$, and $\Delta \phi_{i}(\cdot): \mathfrak{R} \rightarrow \mathfrak{R}$ is the uncertain input nonlinearity satisfying (3.1a)-(3.1b), with the system parameter $a=0.4$ and $q \in N$. Setting the control $u$ as (3.5), by Example 3.1, we have $\|e(t)\|=\|z(t)-x(t)\| \leq k_{1} \cdot e^{-\alpha t}, \forall t \geq 0$. Consequently, by (3.6a)-(3.6f) and (3.7a)-(3.7e), one can see that

$$
\left\|h_{1}(t)-h(t)\right\|=\left\|\phi_{h}(t)-C_{h} z(t)-\phi_{h}(t)+C_{h} x(t)\right\| \leq\left\|C_{h}\right\| \cdot\|e(t)\| \leq k_{1}\left\|C_{h}\right\| e^{-\alpha t}, \quad \forall t \geq 0 .
$$

This implies that one can recover the message $h(t)$ in the receiver system, with the guaranteed exponential convergence rate $\alpha$. In other words, the synchronization of signals $h(t)$ and $h_{1}(t)$ for the proposed secure communication (3.6a)-(3.6f) and (3.7a)-(3.7e) can always be achieved with any prespecified convergence rate $\alpha$. 


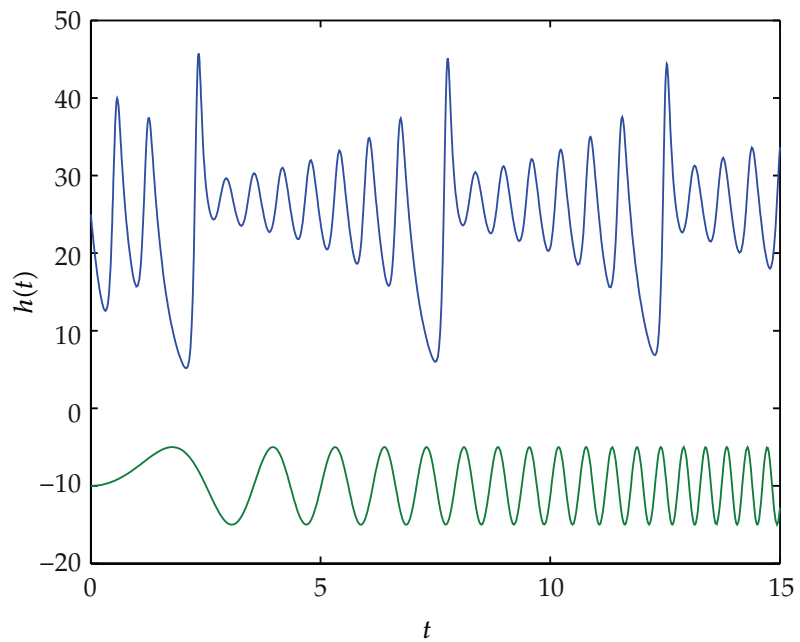

Figure 6: Real message of $h(t) \in \mathfrak{R}^{2 \times 1}$ described in the transmitter of (3.6a)-(3.6f).

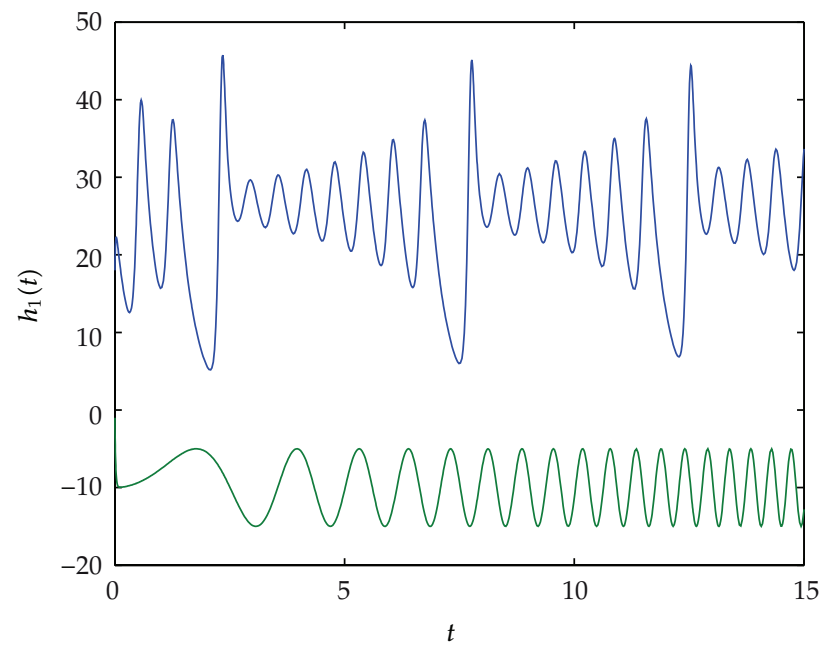

Figure 7: Recoverd message of $h_{1}(t) \in \mathfrak{R}^{2 \times 1}$ described in in the receiver of (3.7a)-(3.7e).

With, for example, $\alpha=6$, the real message $h(t)$, the recovered message $h_{1}(t)$, and the error signal are depicted in Figures 6, 7, and 8, respectively, which clearly indicates that the real message $h(t)$ is recovered after 0.2 seconds.

\section{Conclusion}

In this paper, the notion of practical synchronization has been introduced and the chaos synchronization of master-slave chaotic systems with uncertain input nonlinearities has been investigated. Based on the DII approach, a simple linear control has been proposed to realize the practical synchronization for the master-slave chaotic systems with uncertain input nonlinearities. Furthermore, the guaranteed exponential convergence rate can be 


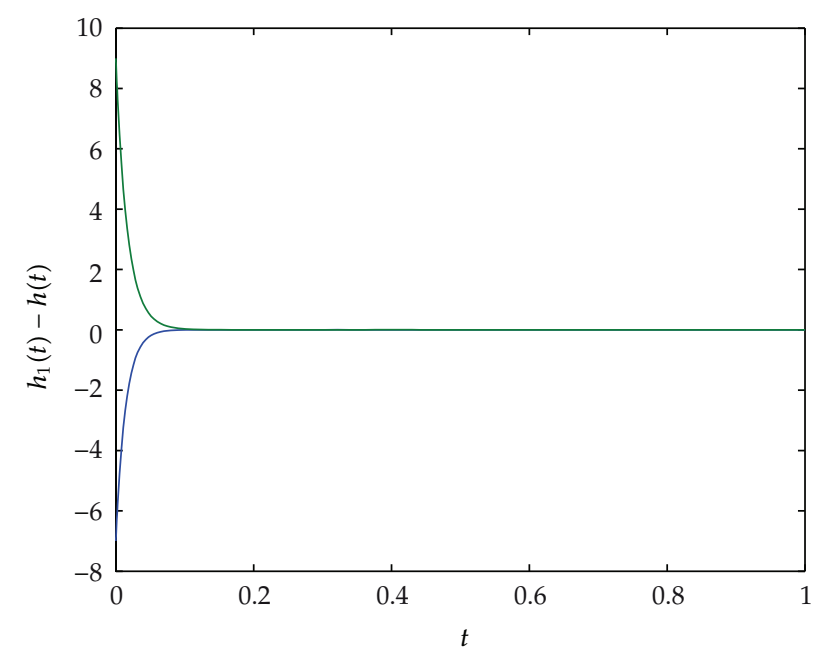

Figure 8: Error signal of $h_{1}(t)-h(t)$.

prespecified. Applications of proposed master-slave chaotic synchronization technique to secure communication as well as several numerical simulations have also been given to demonstrate the feasibility and effectiveness of the obtained result.

\section{Acknowledgment}

The author thanks the National Science Council of Republic of China for supporting this work under Grant NSC-100-2221-E-214-015. The author would also like to thank the Metal Industries Research \& Development Centre for supporting this work under Grant ISU101GOV-37. Furthermore, the author would like to thank anonymous reviewers for their helpful comments.

\section{References}

[1] K. J. Astrom and R. M. Murray, Feedback Systems: An Introduction For Scientists And Engineers, Princeton University Press, Princeton, NJ, USA, 2008.

[2] M. Dhamala and Y. C. Lai, "Controlling transient chaos in deterministic flows with applications to electrical power systems and ecology," Physical Review E, vol. 59, no. 2, pp. 1646-1655, 1999.

[3] P. Dorato, Analytic Feedback System Design: An Interpolation Approach, Brooks/Cole, Pacific Grove, Calif, USA, 1999.

[4] G. F. Franklin, J. D. Powell, and A. Emani-Naeini, Feedback Control of Dynamic Systems, Prentice-Hall, New Jersey, NJ, USA, 2002.

[5] J. Li, W. Li, and Q. Li, "Sliding mode control for uncertain chaotic systems with input nonlinearity," Communications in Nonlinear Science and Numerical Simulation, vol. 17, no. 1, pp. 341-348, 2012.

[6] J. S. Lin and J. J. Yan, "Adaptive synchronization for two identical generalized Lorenz chaotic systems via a single controller," Nonlinear Analysis: Real World Applications, vol. 10, no. 2, pp. 1151-1159, 2009.

[7] Y. J. Sun, "Chaos synchronization of uncertain Genesio-Tesi chaotic systems with deadzone nonlinearity," Physics Letters A, vol. 373, no. 36, pp. 3273-3276, 2009.

[8] X. Wu, G. Chen, and J. Cai, "Chaos synchronization of the master-slave generalized Lorenz systems via linear state error feedback control," Physica D, vol. 229, no. 1, pp. 52-80, 2007. 
[9] W. Xiang and F. Chen, "An adaptive sliding mode control scheme for a class of chaotic systems with mismatched perturbations and input nonlinearities," Communications in Nonlinear Science and Numerical Simulation, vol. 16, no. 1, pp. 1-9, 2011.

[10] C. C. Yang, "One input control for exponential synchronization in generalized Lorenz systems with uncertain parameters," Journal of the Franklin Institute, vol. 349, pp. 349-365, 2012.

[11] H. Yu, J. Wang, B. Deng et al., "Adaptive backstepping sliding mode control for chaos synchronization of two coupled neurons in the external electrical stimulation," Communications in Nonlinear Science and Numerical Simulation, vol. 17, pp. 1344-1354, 2012.

[12] Q. Zhang, J. Lü, and S. Chen, "Coexistence of anti-phase and complete synchronization in the generalized Lorenz system," Communications in Nonlinear Science and Numerical Simulation, vol. 15, no. 10, pp. 3067-3072, 2010.

[13] Y. J. Sun, "Solution bounds of generalized Lorenz chaotic systems," Chaos, Solitons and Fractals, vol. 40, no. 2, pp. 691-696, 2009.

[14] Y. J. Sun, "A simple observer design of the generalized Lorenz chaotic systems," Physics Letters A, vol. 374, no. 7, pp. 933-937, 2010.

[15] Z. Zhang, H. Shao, Z. Wang, and H. Shen, "Reduced-order observer design for the synchronization of the generalized Lorenz chaotic systems," Applied Mathematics and Computation, vol. 218, pp. 7614 7621, 2012.

[16] A. Boulkroune and M. M'Saad, "A fuzzy adaptive variable-structure control scheme for uncertain chaotic MIMO systems with sector nonlinearities and dead-zones," Expert Systems with Applications, vol. 38, pp. 14744-14750, 2011.

[17] D. H. Ji, J. H. Park, and S. C. Won, "Master-slave synchronization of Lur'e systems with sector and slope restricted nonlinearities," Physics Letters A, vol. 373, no. 11, pp. 1044-1050, 2009.

[18] Z. Zhang, H. Shen, and J. Li, "Adaptive stabilization of uncertain unified chaotic systems with nonlinear input," Applied Mathematics and Computation, vol. 218, pp. 4260-4267, 2011.

[19] A. Boulkroune, M. M'Saad, and M. Farza, "Adaptive fuzzy controller for multivariable nonlinear state time-varying delay systems subject to input nonlinearities," Fuzzy Sets and Systems, vol. 164, no. 1, pp. 45-65, 2011.

[20] X. Wu, X. Wu, X. Luo, Q. Zhu, and X. Guan, "Neural network-based adaptive tracking control for nonlinearly parameterized systems with unknown input nonlinearities," Neurocomputing, vol. 82, no. 1, pp. 127-142, 2012.

[21] J. Lü, G. Chen, D. Cheng, and S. Celikovsky, "Bridge the gap between the Lorenz system and the Chen system," International Journal of Bifurcation and Chaos in Applied Sciences and Engineering, vol. 12, no. 12, pp. 2917-2926, 2002. 


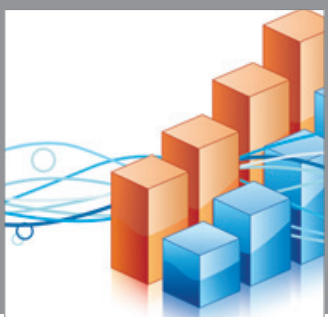

Advances in

Operations Research

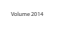

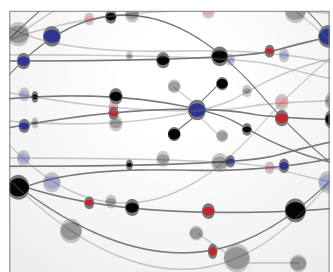

\section{The Scientific} World Journal
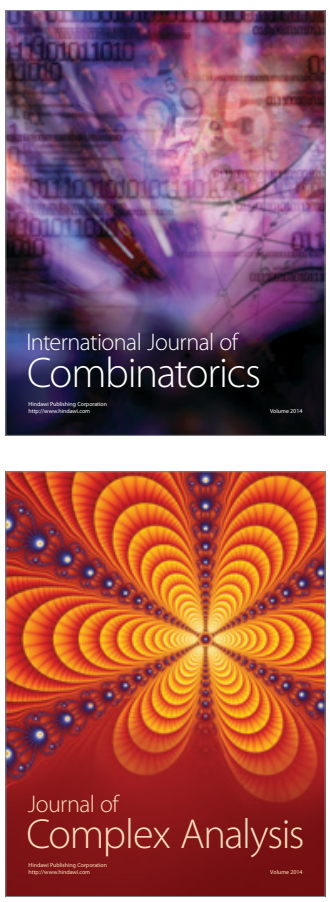

International Journal of

Mathematics and

Mathematical

Sciences
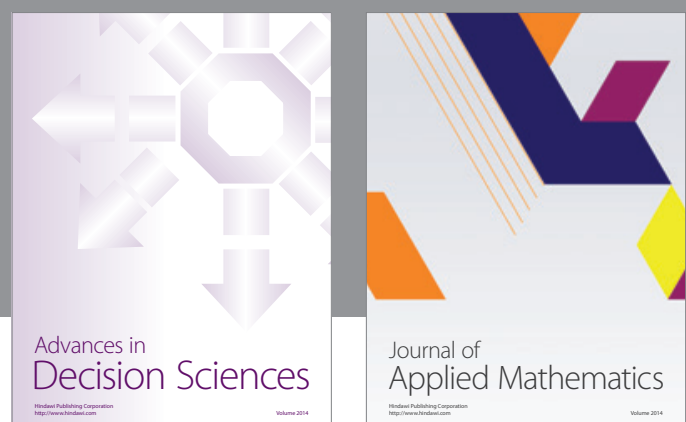

Journal of

Applied Mathematics
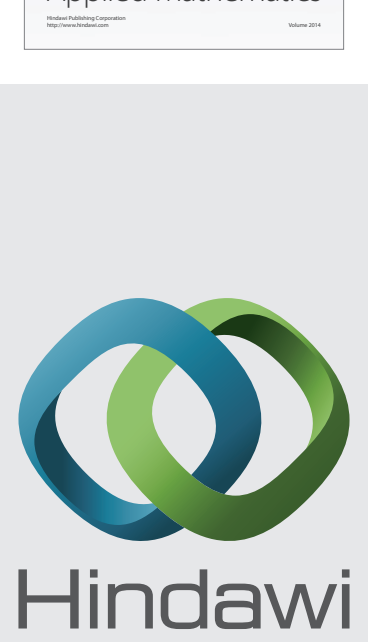

Submit your manuscripts at http://www.hindawi.com
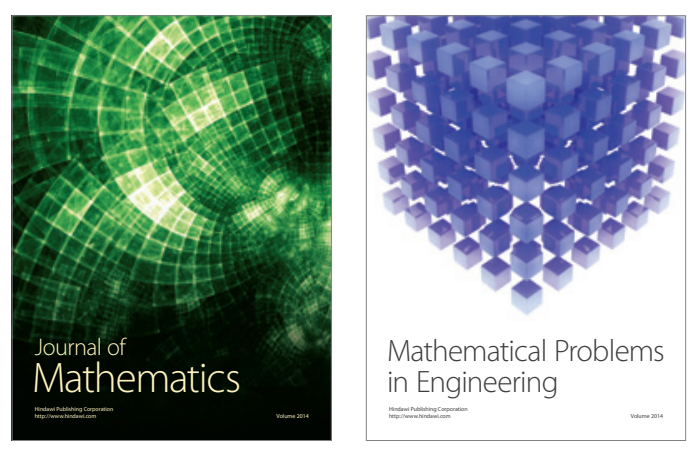

Mathematical Problems in Engineering
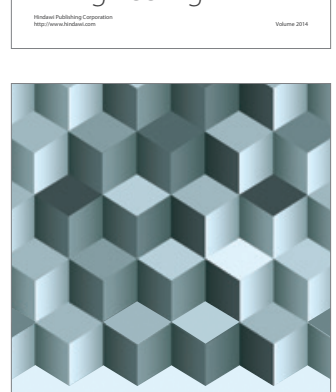

Journal of

Function Spaces
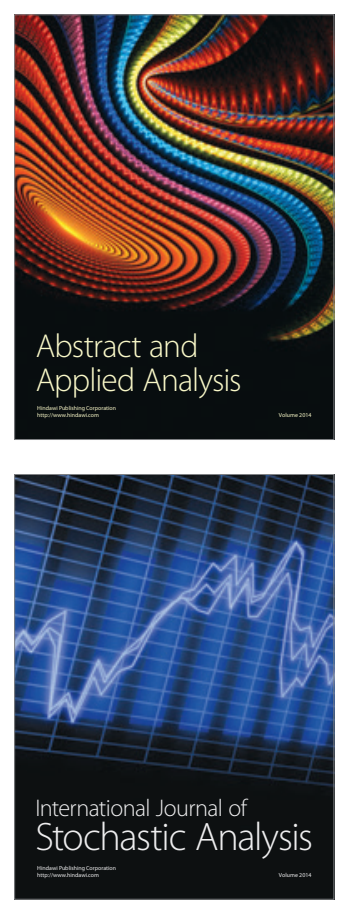

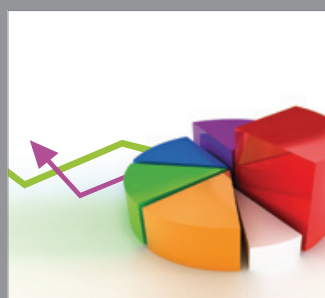

ournal of

Probability and Statistics

Promensencen
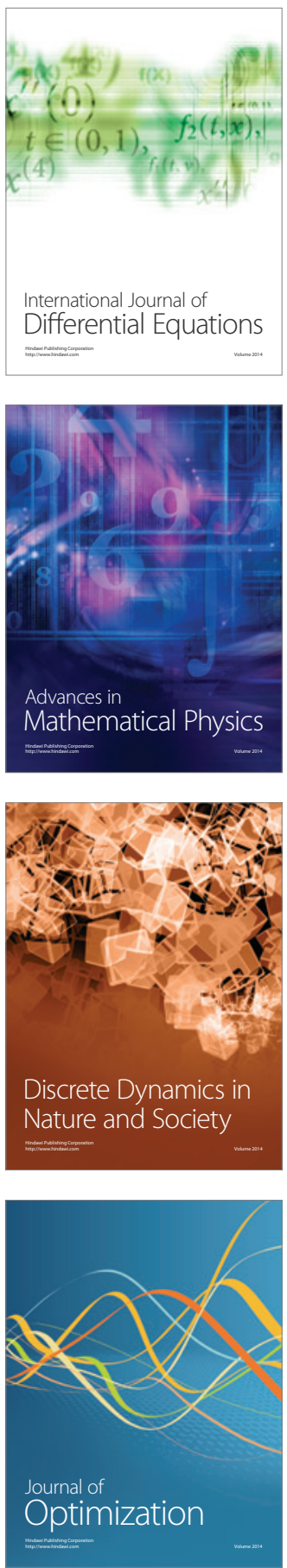